\title{
Breakfast consumption and physical activity in British adolescents
}

\author{
K. Corder $^{1 *}$, E. M. F. van Sluijs ${ }^{1}$, R. M. Steele ${ }^{1}$, A. M. Stephen ${ }^{2}$, V. Dunn ${ }^{3}$, D. Bamber ${ }^{3}$, I. Goodyer ${ }^{3}$, \\ S. J. Griffin ${ }^{1}$ and U. Ekelund ${ }^{1}$ \\ ${ }^{1}$ MRC Epidemiology Unit, Institute of Metabolic Science, Addenbrooke's Hospital, Box 285, Hills Road, Cambridge \\ CB2 OQQ, UK \\ ${ }^{2}$ MRC Human Nutrition Research, Elsie Widdowson Laboratory, 120 Fulbourn Road, Cambridge CB1 9NL, UK \\ ${ }^{3}$ Developmental Psychiatry Section, Department of Psychiatry, University of Cambridge, Douglas House, 18b Trumpington \\ Road, Cambridge CB2 $8 A H$, UK
}

(Received 2 June 2010 - Revised 23 July 2010 - Accepted 28 July 2010 - First published online 1 September 2010)

\section{Abstract}

Studies show an inverse relationship between breakfast frequency and weight gain. This may reflect poor eating habits generally and associated low physical activity (PA) or direct impacts of breakfast on mechanisms leading to lethargy and reduced PA. The relationship between breakfast frequency and PA is inconclusive. We aimed to determine whether breakfast frequency is associated with PA levels in British adolescents independent of body composition and socio-economic status (SES). Habitual breakfast frequency (self-report questionnaire) was assessed in 877 adolescents ( $43 \%$ male, age 14.5 (SD 0.5) years old). PA was measured over $5 \mathrm{~d}$ (accelerometry, average counts/ min; cpm). Associations between daily PA and breakfast frequency were assessed using linear regression adjusted for body fat percentage and SES. Effect modification by sex and associations with PA during the morning (06.00-12.00 hours) were explored. For boys, there were no significant associations between breakfast frequency and PA. For girls, less frequent breakfast consumption was significantly associated with lower PA (cpm) during the morning (occasional $v$. frequent $\beta-6 \cdot 1(95 \%$ CI $-11 \cdot 1,-1 \cdot 1), P=0 \cdot 017)$ when adjusted for body fat percentage and SES. There were no associations between PA and breakfast consumption over the whole day; however, for girls, less frequent breakfast consumption may be associated with lower PA levels during the morning, suggesting that breakfast consumption should perhaps be taken into consideration when aiming to promote PA in adolescent girls.

\section{Key words: Physical activity: Eating behaviour: Cross-sectional studies: Adolescents}

Breakfast is often considered to be the most important meal of the day and skipping breakfast has been associated with overweight ${ }^{(1)}$ and higher BMI in adolescents ${ }^{(2)}$. The majority of studies in a recent review examining breakfast habits and body weight in youth found an association between skipping breakfast and a higher $\mathrm{BMI}^{(3)}$.

Skipping breakfast may be associated with poor diet generally, possibly explaining the association between breakfast consumption frequency (breakfast frequency) and overweight, and it has been shown that skipping breakfast leads to the consumption of more high-fat snacks later in the day ${ }^{(4)}$. However, a recent review concluded that those who ate breakfast more frequently had a higher total energy intake, but were less likely to be overweight than those who skip breakfast ${ }^{(3)}$. Therefore, breakfast consumption may not solely influence body weight via energy intake but also through an effect on energy expenditure. Those who skip breakfast may also be those who do little physical activity (PA), which might, at least in part, account for the association between breakfast frequency and overweight. Another possible explanation is that skipping breakfast could have a causal effect and actually lead to apathy and lethargy and therefore reduced PA throughout the day ${ }^{(5-7)}$. Some studies suggest that physically active adolescents are less likely to skip breakfast and are more likely to regularly eat breakfast than inactive individuals ${ }^{(2,8,9)}$. However, this relationship is inconclusive, with several studies showing no association between breakfast eating behaviours and $\mathrm{PA}^{(10-12)}$. Most of the studies assessing breakfast consumption and PA have used self-reported PA data; therefore, measurement error or bias may partly explain why results vary between studies.

Socio-economic status (SES), body composition and sex are associated with both breakfast consumption and PA. Individuals from low socio-economic backgrounds are

Abbreviations: PA, physical activity; SES, socio-economic status. 
more likely to skip breakfast than those with a higher $\operatorname{SES}^{(3,10,13,14)}$, whereas the relationship between PA and SES is inconclusive but tends towards a positive association $^{(15,16)}$. Several studies have shown that a less favourable body composition is associated with less frequent breakfast consumption ${ }^{(2,8,9)}$ and a lower PA level ${ }^{(17,18)}$. Therefore, consideration of sex, SES and body composition a priori and adequate adjustment for these confounders are important for a thorough investigation of the association between breakfast consumption and PA.

Despite PA and diet arguably being the two most important modifiable risk factors for overweight and obesity, there has been relatively little research investigating the detailed associations between these complex behaviours. Furthermore, research into PA and diet often does not take the other field into account. If missing breakfast is associated with low PA levels, it is unclear whether the lower levels of PA are restricted to the morning or persist throughout the day.

The ROOTS study incorporates an objective measure of PA in a large population-based sample of British adolescents and is well placed to investigate the relationship between breakfast frequency and PA. We aimed to determine whether breakfast frequency is associated with PA levels in British adolescents independent of body composition and SES and whether the association differs in boys and girls.

\section{Methods}

\section{Participants}

Volunteers consisted of 877 boys ( $n$ 379) and girls ( $n$ 498), recruited to take part in the ROOTS study ${ }^{(19)}$. The ROOTS study is a longitudinal cohort study established to determine the relative contributions that specific genetic, physiological, psychological and social variables make to the overall risk of psychopathology during adolescence. The study recruited 1238 adolescents through schools in the counties of Cambridgeshire and Suffolk, UK. Teenagers were recruited into the study at age 14 years to be reassessed after 18 months and 3 years with repeat measures of PA, diet and mental health.

For the baseline data collection, secondary schools from Cambridgeshire and Suffolk, UK, the surrounding villages and the area extending 30 miles north, 20 miles south and 20 miles west of Cambridge city were approached. Of the twenty-seven schools invited, eighteen agreed to participate. Study information, invitation letters and parent and student consent forms were sent to eligible parents and students via eighteen Cambridgeshire and Suffolk secondary schools (sixteen state and two independent); those completing these forms were invited to the baseline part of the study. A second information sheet and consent form regarding the $\mathrm{PA}$ and diet assessment phase of the study were sent to those attending the baseline assessment and those returning the completed consent forms were invited to a measurement session at their school. The present study used data from this second assessment approximately 6 months after the baseline visit, which examined PA, diet and body composition and was carried out between November 2005 and July 2007. In total, 1200 ROOTS baseline participants were invited to the PA assessment part of the study. All procedures were verbally explained and participants could choose to decline all or any part of the study at any stage. The full ROOTS study was approved by the Cambridge Local Research Ethics Committee.

\section{Measurements}

Volunteers were visited at school by a team of trained researchers. Height was measured to the nearest $0 \cdot 1 \mathrm{~cm}$ (Leicester Height Metre; Invicta Plastics, Leicester, UK), weight was measured to the nearest $0 \cdot 1 \mathrm{~kg}$ (Tanita TBF300 MA, Tanita, Tokyo, Japan), in light clothing and without shoes and socks. Fat-free mass and fat mass were determined using bio-electrical impedance (Tanita TBF300 MA). Previously validated and published equations were used to derive body fat percentage ${ }^{(20)}$.

Breakfast frequency was assessed using a self-report questionnaire, with the question: 'How many days a week do you usually eat breakfast?' Response categories were 'rarely or never', ' 1 to 2 ', ' 3 to 4 ' or ' 5 or more'; the middle two categories were combined for analysis and therefore participants were categorised as either 'never', 'occasional' (1-4 times a week) or 'frequent' ( $\geq$ five times a week) breakfast consumers. This question was self-reported during the testing session at school.

PA was objectively assessed using the Actiheart combined heart rate and movement sensor (Cambridge Neurotechnology, Papworth, UK). The monitor has been validated to assess PA in youth ${ }^{(21,22)}$ and was attached to the torso with two standard electrocardiogram electrodes and set to record in $30 \mathrm{~s}$ epochs, the shortest epoch allowing for five consecutive days of recording. Volunteers were instructed to wear the monitor continuously, including during sleep and water-based activities for four consecutive days from the testing session, always including a complete weekend. Participants were asked to return the monitors to school approximately 1 week after the visit, where they were collected by the researchers. The PA data were expressed as overall PA and were expressed as an average value for each individual (mean daily counts/ $\mathrm{min}$ ). We used the accelerometry data as a continuous variable in the raw form because we hypothesised that lack of breakfast could lead to apathy and lethargy and we were interested in all movement including habitual PA. Consequently, we used only the accelerometry data in this analysis, as heart rate monitoring has limitations for the assessment of light-intensity habitual PA ${ }^{(23)}$. Accelerometry data were analysed using a batch processing program 
(www.mrc-epid.cam.ac.uk/Research/PA/Downloads.html). When $20 \mathrm{~min}$ of consecutive zeros were present in the accelerometry data, they were removed and it was assumed that the monitor was unworn at that time; consequently, all the days consisting of over $500 \mathrm{~min}$ of valid data were included in the analysis. Volunteers were excluded if they did not have at least one complete day of recording.

The 'A classification of residential neighbourhoods' (ACORN) index is used here as a proxy for area-level SES. The ACORN index is a postcode-based classification system, which categorises all UK postcodes into one of five categories using 125 demographic and 287 lifestyle variables $^{(24)}$. From high to low SES, these categories are wealthy achievers, urban prosperity, comfortably off, moderate means and hard-pressed. Where postcodes were missing and SES data were therefore unavailable ( $n$ 10), the average score for the school was used.

\section{Statistical analyses}

Analyses were carried out using Stata 10 (Statacorp, Lakeway Drive, College Station, TX, USA). Sex differences in descriptive characteristics, breakfast frequency and PA were assessed using Student's $t$ tests or $\chi^{2}$ tests depending on the nature of the variable. The analyses were stratified by sex, as the majority of studies show that girls are significantly more likely to skip breakfast than boys ${ }^{(2,3,7,13,25)}$ and that boys are more physically active than girls ${ }^{(15,16)}$. PA $(P=0 \cdot 13)$ and breakfast frequency $(P=0 \cdot 46)$ did not differ by school; so, analyses were not adjusted for school clustering.

Linear regression analysis was used to investigate the associations between PA and breakfast frequency. Firstly, unadjusted models were examined, and then the two sets of adjusted models; the first including SES and the second including SES and body fat percentage. These analyses were carried out with mean daily PA and for PA during the morning (06.00-12.00 hours).

No differences were found between analyses carried out separately for weekday and weekend days. Therefore, the PA variable used here was derived as an average over both weekend and weekdays.

\section{Results}

Of the 1200 adolescents invited into the study, 998 (83\%) adolescents and parents completed postal informed consent and 931 (93\% of those consenting) attended a testing session at school. There was no significant difference in SES $(P=0 \cdot 60)$ or sex $(P=0 \cdot 11)$ between those taking part in only the baseline assessment in comparison and those also measured in the PA and diet assessment. Data from 877 (88\% of those consenting and $94 \%$ of those attending a testing session) adolescents with complete body composition, PA and diet measurements were included in the analyses presented here.

Descriptive characteristics of the participants are summarised in Table 1. Approximately, $70 \%$ of the participants ate breakfast frequently, $21 \%$ ate breakfast occasionally and $9 \%$ never ate breakfast. Breakfast frequency differed by sex, with approximately $6 \%$ of boys never eating breakfast compared with about $12 \%$ of the girls $(P<0.001)$ and boys were significantly more active than the girls $(P<0.001)$. Compared with those with complete data, those with missing PA or dietary data did not significantly differ by sex $(P=0 \cdot 26)$, body weight $(P=0 \cdot 86)$, body fat $(P=0 \cdot 37)$ or SES $(P=0.97)$.

Table 1. Participant characteristics

(Mean values and standard deviations*)

\begin{tabular}{|c|c|c|c|c|c|c|c|}
\hline & \multicolumn{2}{|c|}{ All $(n 877)$} & \multicolumn{2}{|c|}{ Boys ( $n$ 379) } & \multicolumn{2}{|c|}{ Girls ( $n$ 498) } & \multirow[b]{2}{*}{$P$ value for difference } \\
\hline & Mean & SD & Mean & SD & Mean & SD & \\
\hline Age (years) & 14.5 & 0.5 & 14.5 & 0.5 & 14.5 & 0.5 & $0 \cdot 17$ \\
\hline Wt $(\mathrm{kg})$ & $57 \cdot 6$ & $11 \cdot 0$ & 59.9 & 11.5 & 55.9 & $10 \cdot 2$ & $<0.001$ \\
\hline Height $(\mathrm{cm})$ & $166 \cdot 4$ & $7 \cdot 8$ & $171 \cdot 1$ & $7 \cdot 6$ & $162 \cdot 9$ & 5.9 & $<0.001$ \\
\hline Body fat (\%) & $19 \cdot 5$ & $9 \cdot 6$ & $12 \cdot 2$ & $6 \cdot 7$ & $25 \cdot 1$ & $7 \cdot 6$ & $<0.001$ \\
\hline SES $(n, \%) \dagger$ & & & & & & & 0.37 \\
\hline Wealthy achievers & 492 & $56 \cdot 1$ & 206 & $54 \cdot 3$ & 286 & 57.5 & \\
\hline Urban prosperity & 58 & $6 \cdot 6$ & 31 & $8 \cdot 2$ & 27 & 5.4 & \\
\hline Comfortably off & 201 & $22 \cdot 9$ & 83 & 21.9 & 118 & $23 \cdot 7$ & \\
\hline Moderate means & 40 & $4 \cdot 6$ & 17 & 4.5 & 23 & $4 \cdot 6$ & \\
\hline Hard pressed & 86 & $9 \cdot 8$ & 42 & $11 \cdot 1$ & 44 & $8 \cdot 8$ & \\
\hline $\begin{array}{l}\text { Physical activity (counts/min) } \\
\text { Breakfast frequency }(n, \%)\end{array}$ & $55 \cdot 8$ & $26 \cdot 4$ & 64.5 & $32 \cdot 4$ & $48 \cdot 7$ & $17 \cdot 8$ & $\begin{array}{l}<0.001 \\
<0.001\end{array}$ \\
\hline Never & 84 & $9 \cdot 6$ & 22 & $5 \cdot 8$ & 62 & $12 \cdot 5$ & \\
\hline Occasional & 184 & $21 \cdot 0$ & 65 & $17 \cdot 2$ & 119 & 23.9 & \\
\hline Frequent & 609 & $69 \cdot 4$ & 292 & $77 \cdot 0$ & 317 & $63 \cdot 6$ & \\
\hline
\end{tabular}

SES, socio-economic status.

*Unless otherwise stated.

†Derived from home postcode (a classification of residential neighbourhoods category) scored highest SES (1) to lowest SES (5).

$\ddagger$ Mean daily physical activity. 
Table 2. Simple and adjusted associations between breakfast consumption frequency and physical activity ${ }^{\star}$ (Mean values and $95 \%$ confidence intervals)

\begin{tabular}{|c|c|c|c|c|c|c|c|c|c|}
\hline \multirow[b]{2}{*}{ Breakfast frequency } & \multicolumn{3}{|c|}{ Unadjusted } & \multicolumn{3}{|c|}{ Model $1 \dagger$} & \multicolumn{3}{|c|}{ Model $2 \ddagger$} \\
\hline & $\beta$ & $95 \% \mathrm{Cl}$ & $P$ & $\beta$ & $95 \% \mathrm{Cl}$ & $P$ & $\beta$ & $95 \% \mathrm{Cl}$ & $P$ \\
\hline \multicolumn{10}{|c|}{ Average counts/min (reference category: frequent breakfast consumer) } \\
\hline \multicolumn{10}{|c|}{ Boys $(n$ 379) } \\
\hline \multicolumn{10}{|l|}{ Morning } \\
\hline Never & -2.9 & $-20 \cdot 8,14 \cdot 9$ & 0.75 & $-4 \cdot 8$ & $-22 \cdot 6,13 \cdot 1$ & 0.60 & $-5 \cdot 3$ & $-23 \cdot 2,12 \cdot 5$ & 0.56 \\
\hline Occasional & $6 \cdot 0$ & $-4 \cdot 98,17 \cdot 0$ & 0.28 & $5 \cdot 0$ & $-5 \cdot 9,15 \cdot 9$ & 0.37 & $4 \cdot 2$ & $-6 \cdot 7,15 \cdot 2$ & 0.45 \\
\hline \multicolumn{10}{|l|}{ Whole day } \\
\hline Never & -2.6 & $-16 \cdot 7,11.5$ & 0.72 & $-4 \cdot 0$ & $-18 \cdot 1,10 \cdot 1$ & 0.58 & $-4 \cdot 0$ & $-18 \cdot 2,10 \cdot 1$ & 0.58 \\
\hline Occasional & $4 \cdot 1$ & $-4 \cdot 6,12 \cdot 8$ & 0.36 & 3.5 & $-5 \cdot 2,12 \cdot 3$ & 0.43 & 3.5 & $-5 \cdot 3,9 \cdot 2$ & 0.43 \\
\hline \multicolumn{10}{|l|}{ Girls ( $n$ 498) } \\
\hline \multicolumn{10}{|l|}{ Morning } \\
\hline Never & -3.8 & $-10 \cdot 2,2 \cdot 5$ & 0.69 & -4.4 & $-10 \cdot 8,12 \cdot 0$ & 0.18 & -4.5 & $-11 \cdot 0,1.9$ & 0.17 \\
\hline Occasional & $-4 \cdot 1$ & $-9 \cdot 0,0 \cdot 8$ & $0 \cdot 10$ & -5.9 & $-10 \cdot 9,-1.0$ & 0.020 & $-6 \cdot 1$ & $-11 \cdot 1,-1 \cdot 1$ & 0.017 \\
\hline \multicolumn{10}{|l|}{ Whole day } \\
\hline Never & 1.5 & $-3 \cdot 4,6 \cdot 3$ & 0.56 & 0.86 & $-4 \cdot 0,5 \cdot 8$ & 0.73 & 0.70 & $-4 \cdot 2,5 \cdot 6$ & 0.78 \\
\hline Occasional & -1.0 & $-4 \cdot 8,2 \cdot 7$ & 0.59 & $-2 \cdot 1$ & $-5 \cdot 9,1 \cdot 8$ & 0.30 & $-2 \cdot 4$ & $-6 \cdot 2,1 \cdot 5$ & 0.23 \\
\hline
\end{tabular}

SES, socio-economic status.

* Mean daily counts/min.

† Model 1 adjusted for SES.

$\ddagger$ Model 2 adjusted for SES and body fat percentage.

Table 2 shows a summary of PA data by breakfast group. There were no significant differences in unadjusted analyses in PA by breakfast group over the whole day or morning for either boys or girls.

There were no significant associations between breakfast frequency and PA over the whole day and no associations were observed for boys. For girls, during the morning, those who were occasional consumers of breakfast had a significantly lower PA (Table 2) than those who were frequent breakfast consumers when adjusted for SES. This association was not attenuated by the addition of body fat percentage to the model.

\section{Discussion}

The present results suggest that overall daily PA is not associated with breakfast consumption in British adolescents. However, less frequent breakfast consumption was associated with lower PA during the morning for girls, independent of body composition and SES. These results indicate that the consumption of breakfast might influence the PA differently across the day and further investigation is warranted. This may be because skipping breakfast leads to apathy and lethargy in the morning ${ }^{(5-7)}$, but then the effect may weaken, possibly influenced by later meals and non-dietary factors.

Fewer boys than girls never ate breakfast and this more homogeneous outcome may have contributed to the lack of association in boys ${ }^{(26)}$. Additionally, participants were not provided with a definition of breakfast; consequently, boys and girls may have had different opinions of what constitutes breakfast, and different factors may influence this association in boys and girls. Further, the question used to assess breakfast consumption was not validated; comparison against a diet diary would help to ascertain the accuracy of this question. Our finding of a significant relationship between breakfast eating and PA in girls and not in boys concurs with previous studies that have shown stronger associations between breakfast skipping and unhealthy behaviours in girls than boys ${ }^{(2,3,7,13,25)}$. It is also possible that mood difficulties or depression could play a role, as appetite loss and lethargy are both associated with depression, and mood difficulties are significantly greater in girls than in boys ${ }^{(27)}$.

The association between breakfast frequency and PA became significant when adjusted for SES. This is likely to be due to negative confounding ${ }^{(28)}$, as higher SES is associated with higher PA $(P=0.04)$ and higher SES is also associated with a higher breakfast frequency $(P=0.04)$. Therefore, if SES is not accounted for when investigating the association between breakfast frequency and PA, then it is likely that the true association will be underestimated.

None of the significant associations observed in the present study was attenuated by the addition of body fat percentage, suggesting that the association between breakfast consumption and PA is independent of body composition. This adds support to the hypothesis that breakfast consumption may directly influence PA and may have an impact on overweight via PA, rather than, or, in addition to energy intake. However, this is a cross-sectional study, so we cannot imply causation from these results.

There are a few studies assessing the association between dietary behaviours and PA using objective PA data $^{(11,29)}$, but, to our knowledge, this is the first study examining the association between breakfast frequency and objectively measuring PA in adolescents. Two previous studies using objective monitoring to assess PA and diet have shown conflicting results, with one showing that PA and fat consumption were inversely related ${ }^{(29)}$ and the 
other indicating no relationship between change in diet or $\mathrm{PA}^{(11)}$.

The relatively small number of participants who never ate breakfast (9\%) is a limitation of the present study, but is similar to other studies in adolescents. A Finnish study on 16 -year-old boys and girls showed that $16 \%$ of boys and $13 \%$ of girls never ate breakfast ${ }^{(2)}$, with an American study finding that $3.6 \%$ of children rarely ate breakfast ${ }^{(1)}$, suggesting that this may be an unavoidable limitation when examining breakfast frequency. Another study design, such as a case-control study, might be necessary to gain sufficient power to adequately investigate this research question and this may also imply that when investigating breakfast skipping, more precise measures are needed. One possible reason for lower PA levels among 'occasional', but not for 'never' breakfast consumers may be a lack of power, as relatively few boys reported never eating breakfast. Day-by-day PA and breakfast data would be useful to further explore the PA levels of these 'never' breakfast consumers and it would be interesting to examine the characteristics of these participants, including the potential clustering of other health behaviours in this group. An important point to note is that this would be much less of a limitation for examination of breakfast consumption and PA on a day-by-day basis, as $20 \%$ of adolescents in the present study were occasional breakfast consumers and therefore ate breakfast one to four times a week.

The objective measurement of PA used in all participants is a strength of the present study as it allows comparison between different breakfast frequency groups without any recall bias in the PA measurement. The breakfast variable used here assessed habitual breakfast frequency with one question, not on a day-by-day basis, and not accounting for what the adolescents ate. It is also possible that interpretations of 'breakfast' differ, especially in response to a global question ${ }^{(30)}$ and may differ between boys and girls, which could contribute to the differences seen here and in other studies ${ }^{(2,3,7,13,25)}$. As our breakfast variable assesses habitual breakfast consumption, it is possible that whether participants did or did not eat breakfast on measurement days may have influenced their answer. Furthermore, for the occasional breakfast consumers, we cannot be certain whether they were more active on the days when they ate breakfast or whether they ate breakfast on the days on which their PA was measured. These results represent a cross-sectional analysis of data embedded within the ROOTS longitudinal study and this analysis would have been more powerful if breakfast consumption had been measured at baseline and PA at follow-up. Unfortunately, although the present study was longitudinal for some factors, only PA and breakfast consumption were assessed in all the participants at this time point.

We cannot exclude the possibility that the association between breakfast frequency and PA during the morning in girls could be due to a clustering of healthy behaviours rather than a direct association between breakfast consumption and $\mathrm{PA}^{(2,31,32)}$. It has been shown that breakfast skipping is associated with smoking and frequent alcohol consumption in adolescents and with body dissatisfaction, dieting and disordered eating in adolescent girls ${ }^{(2)}$. The classification of SES used here is a proxy measure derived from individual postcodes in five categories and we must acknowledge that our finding relies on adjustment for this confounding variable. It would have been preferable to include an individual-level SES variable such as parental education, rather than the neighbourhood-level SES variable used; unfortunately, individual-level SES data were unavailable. This may also be important, as it is possible that different SES variables may show different associations with PA and potentially with breakfast consumption. Body composition data indicated that this sample had slightly lower levels of overweight and obesity ( $27 \cdot 2 \%$ overweight and obese) than those from the East of England of a similar age $(34 \cdot 1 \%)^{(33)}$.

In the final adjusted model, girls who only occasionally ate breakfast did $6 \cdot 1$ counts/min less PA over the morning than those who frequently ate breakfast. This equates to approximately $12.5 \%$ of girl's average daily PA values and an increase in PA of this magnitude would be substantial.

The importance of education promoting regular breakfast eating to children, adolescents and their parents and recommendations for the introduction of school breakfast programmes have been stated as important targets for reduce obesity in youth ${ }^{(10,34)}$. The present results add weight to the arguments that these health promotion strategies may warrant further investigation and may prove important in programmes aiming to increase PA, especially those targeted at girls. Further research is necessary to determine whether the reduction of PA during the morning for girls would be a sufficient justification for breakfast promotion, as although compensated for during the rest of the day, this may lead to other negative health outcomes such as lower cognitive functioning or poorer mood. Further examination of the relationship between breakfast consumption and PA in more detail is potentially important for informing interventions aiming to increase PA and reducing overweight in youth.

\section{Conclusions}

Habitual breakfast frequency was not associated with overall daily PA levels. However, more frequent breakfast consumption may be associated with higher PA levels during the morning for girls; breakfast consumption should perhaps be taken into consideration when aiming to promote PA in adolescent girls.

\section{Acknowledgements}

The authors acknowledge Charlotte Ridgway, Debra Russo, Kate Westgate, Alice Tompson, Lisa Purslow and Cheryl Kidney for their help with data collection and 
Stephen Sharp for statistical support. We are also grateful to all schools and volunteers who participated in the ROOTS study. ROOTS data collection was supported by the Wellcome Trust (grant no. 074296/Z/04/Z), Medical Research Council Epidemiology Unit and Medical Research Council Human Nutrition Research. Study design, study coordination and data collection for the present study were conducted by D. B., I. G., V. D., U. E., K. C., R. M. S., A. M. S. and E. M. Fv. S; the diet questionnaire was compiled by A. M. S.; data collection was supervised by I. G. and U. E.; K. C. performed the data analyses and drafted the manuscript. All the authors provided critical input on the data analyses and all versions of the manuscript; all the authors approved the final version. None of the authors had any conflicts of interest.

\section{References}

1. Berkey CS, Rockett HR, Gillman MW, et al. (2003) Longitudinal study of skipping breakfast and weight change in adolescents. Int J Obes Relat Metab Disord 27, 1258-1266.

2. Keski-Rahkonen A, Kaprio J, Rissanen A, et al. (2003) Breakfast skipping and health-compromising behaviors in adolescents and adults. Eur J Clin Nutr 57, 842-853.

3. Rampersaud GC, Pereira MA, Girard BL, et al. (2005) Breakfast habits, nutritional status, body weight, and academic performance in children and adolescents. J Am Diet Assoc 105, 743-760 (quiz 761-762).

4. Resnicow K (1991) The relationship between breakfast habits and plasma cholesterol levels in schoolchildren. $J$ Sch Health 61, 81-85.

5. Meyers A (1989) Undernutrition, hunger and learning in children. Nutr News 52, 5-7.

6. Pollitt E \& Mathews R (1998) Breakfast and cognition: an integrative summary. Am J Clin Nutr 67, 804S-813S.

7. Shaw ME (1998) Adolescent breakfast skipping: an Australian study. Adolescence 33, 851-861.

8. Mensink GB, Loose N \& Oomen CM (1997) Physical activity and its association with other lifestyle factors. Eur $J$ Epidemiol 13, 771-778.

9. Magnusson MB, Hulthen L \& Kjellgren KI (2005) Obesity, dietary pattern and physical activity among children in a suburb with a high proportion of immigrants. J Hum Nutr Diet 18, 187-194.

10. Utter J, Scragg R, Mhurchu CN, et al. (2007) At-home breakfast consumption among New Zealand children: associations with body mass index and related nutrition behaviors. $J \mathrm{Am}$ Diet Assoc 107, 570-576.

11. Thompson D, Jago R, Baranowski T, et al. (2004) Covariability in diet and physical activity in African-American girls. Obes Res 12, S46-S54.

12. Mota J, Fidalgo F, Silva R, et al. (2008) Relationships between physical activity, obesity and meal frequency in adolescents. Ann Hum Biol 35, 1-10.

13. Cornelius LJ (1991) Health habits of school-age children. $J$ Health Care Poor Underserved 2, 374-395.

14. Delva J, O'Malley PM \& Johnston LD (2006) Racial/ethnic and socioeconomic status differences in overweight and health-related behaviors among American students: national trends 1986-2003. J Adolesc Health 39, 536-545.
15. Sallis JF, Prochaska JJ \& Taylor WC (2000) A review of correlates of physical activity of children and adolescents. Med Sci Sports Exerc 32, 963-975.

16. van der Horst K, Chin A Paw M, Twisk J, et al. (2007) A brief review on correlates of physical activity and sedentariness in youth. Med Sci Sports Exerc 39, 1241-1250.

17. Janssen I, Katzmarzyk P, Boyce W, et al. (2004) Overweight and obesity in Canadian adolescents and their associations with dietary habits and physical activity patterns. $J$ Adolesc Health 35, 360-367.

18. Sallis JF, Prochaska JJ, Taylor WC, et al. (1999) Correlates of physical activity in a national sample of girls and boys in grades 4 through 12. Health Psychol 18, 410-415.

19. Goodyer IM, Croudace T, Dunn V, et al. (2009) Cohort profile: risk patterns and processes for psychopathology emerging during adolescence: the ROOTS project. Int $J$ Epidemiol 39, 361-369.

20. Tyrrell VJ, Richards G, Hofman P, et al. (2001) Foot-to-foot bioelectrical impedance analysis: a valuable tool for the measurement of body composition in children. Int $J$ Obes Relat Metab Disord 25, 273-278.

21. Corder K, Brage S, Mattocks C, et al. (2007) Comparison of two methods to assess PAEE during six activities in children. Med Sci Sports Exerc 39, 2180-2188.

22. Corder K, Brage S, Wareham NJ, et al. (2005) Comparison of PAEE from combined and separate heart rate and movement models in children. Med Sci Sports Exerc 37, 1761-1767.

23. Livingstone MB, Robson PJ \& Totton M (2000) Energy expenditure by heart rate in children: an evaluation of calibration techniques. Med Sci Sports Exerc 32, 1513-1519.

24. CACI (2009) ACORN User Guide. http://www.caci.co.uk/ acorn2009/CACI.htm (accessed May 2010).

25. Sjoberg A, Hallberg L, Hoglund D, et al. (2003) Meal pattern, food choice, nutrient intake and lifestyle factors in The Goteborg Adolescence Study. Eur J Clin Nutr 57, 1569-1578.

26. Kirkwood B \& Sterne J (2003) Medical Statistics. Oxford: Blackwell Publishing Ltd.

27. Poli P, Sbrana B, Marcheschi M, et al. (2003) Self-reported depressive symptoms in a school sample of Italian children and adolescents. Child Psychiatry Hum Dev 33, 209-226.

28. Hennekens C \& Buring J (1987) Epidemiology in Medicine, 1st ed. Philadelphia, PA: Lippincott Williams and Wilkins.

29. Jago R, Baranowski J, Yoo S, et al. (2004) Relationship between physical activity and diet among African-American girls. Obes Res 12, 55S-63S

30. Albertson AM, Franko DL, Thompson D, et al. (2007) Longitudinal patterns of breakfast eating in black and white adolescent girls. Obesity (Silver Spring) 15, 2282-2292.

31. Affenito SG, Thompson DR, Barton BA, et al. (2005) Breakfast consumption by African-American and white adolescent girls correlates positively with calcium and fiber intake and negatively with body mass index. J Am Diet Assoc 105 938-945.

32. Siega-Riz AM, Popkin BM \& Carson T (2000) Differences in food patterns at breakfast by sociodemographic characteristics among a nationally representative sample of adults in the United States. Prev Med 30, 415-424.

33. The Information Centre (2006) Statistics on Obesity, Physical Activity and Diet: England, 2006. http://www.ic.nhs.uk/ statistics-and-data-collections/health-and-lifestyles/physicalactivity (accessed November 2007).

34. Affenito SG (2007) Breakfast: a missed opportunity. J Am Diet Assoc 107, 565-569. 